\title{
Cloud thermodynamic phase inferred from merged POLDER and MODIS data
}

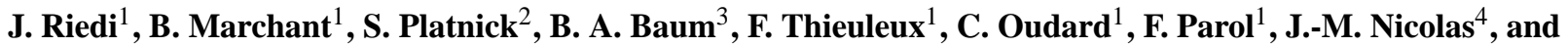 \\ P. Dubuisson ${ }^{1}$ \\ ${ }^{1}$ Laboratoire d'Optique Atmosphérique, UMR 8518, Université de Lille 1 - Sciences et Technologies, CNRS, France \\ ${ }^{2}$ NASA Goddard Space Flight Center, MD, USA \\ ${ }^{3}$ SSEC, University of Wisconsin-Madison, WI, USA \\ ${ }^{4}$ ICARE Data and Services Center, Université des Sciences et Technologies de Lille, France
}

Received: 20 September 2007 - Published in Atmos. Chem. Phys. Discuss.: 4 October 2007

Revised: 12 November 2010 - Accepted: 29 November 2010 - Published: 13 December 2010

\begin{abstract}
The global spatial and diurnal distribution of cloud properties is a key issue for understanding the hydrological cycle, and critical for advancing efforts to improve numerical weather models and general circulation models. Satellite data provides the best way of gaining insight into global cloud properties. In particular, the determination of cloud thermodynamic phase is a critical first step in the process of inferring cloud optical and microphysical properties from satellite measurements. It is important that cloud phase be derived together with an estimate of the confidence of this determination, so that this information can be included with subsequent retrievals (optical thickness, effective particle radius, and ice/liquid water content).

In this study, we combine three different and well documented approaches for inferring cloud phase into a single algorithm. The algorithm is applied to data obtained by the MODIS (MODerate resolution Imaging Spectroradiometer) and POLDER3 (Polarization and Directionality of the Earth Reflectance) instruments. It is shown that this synergistic algorithm can be used routinely to derive cloud phase along with an index that helps to discriminate ambiguous phase from confident phase cases.

The resulting product provides a semi-continuous index ranging from confident liquid to confident ice instead of the usual discrete classification of liquid phase, ice phase, mixed phase (potential combination of ice and liquid particles), or simply unknown phase clouds. The index value provides si-
\end{abstract}

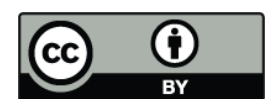

Correspondence to: J. Riedi (jerome.riedi@univ-lille1.fr) multaneously information on the phase and the associated confidence. This approach is expected to be useful for cloud assimilation and modeling efforts while providing more insight into the global cloud properties derived from satellite data.

\section{Introduction}

Clouds are important modulators of the Earth's radiation budget and hydrological cycle. Their macrophysical, microphysical, and optical properties (cloud pressure, temperature, height, optical thickness, thermodynamic phase, effective particle size) and their variation in space and time need to be understood to improve general circulation and weather prediction models. Additionally, cloud properties are being used increasingly in nowcasting activities as part of data assimilation efforts. For nowcasting, the cloud thermodynamic phase is an important indicator used by forecasters to determine hazardous road conditions because occurence of supercooled precipitation strongly increases the risk of ice formation on the ground (Tremblay and Glazer, 2000).

The determination of cloud thermodynamic phase is critical for inferring optical thickness and particle size because ice and water clouds have very different scattering and absorption properties. The quality of the retrieval depends on the ability to match pre-computed radiative transfer calculations with measurements. It is therefore critical that cloud phase be derived together with an estimate of its confidence to help decide which optical models should be used

Published by Copernicus Publications on behalf of the European Geosciences Union. 


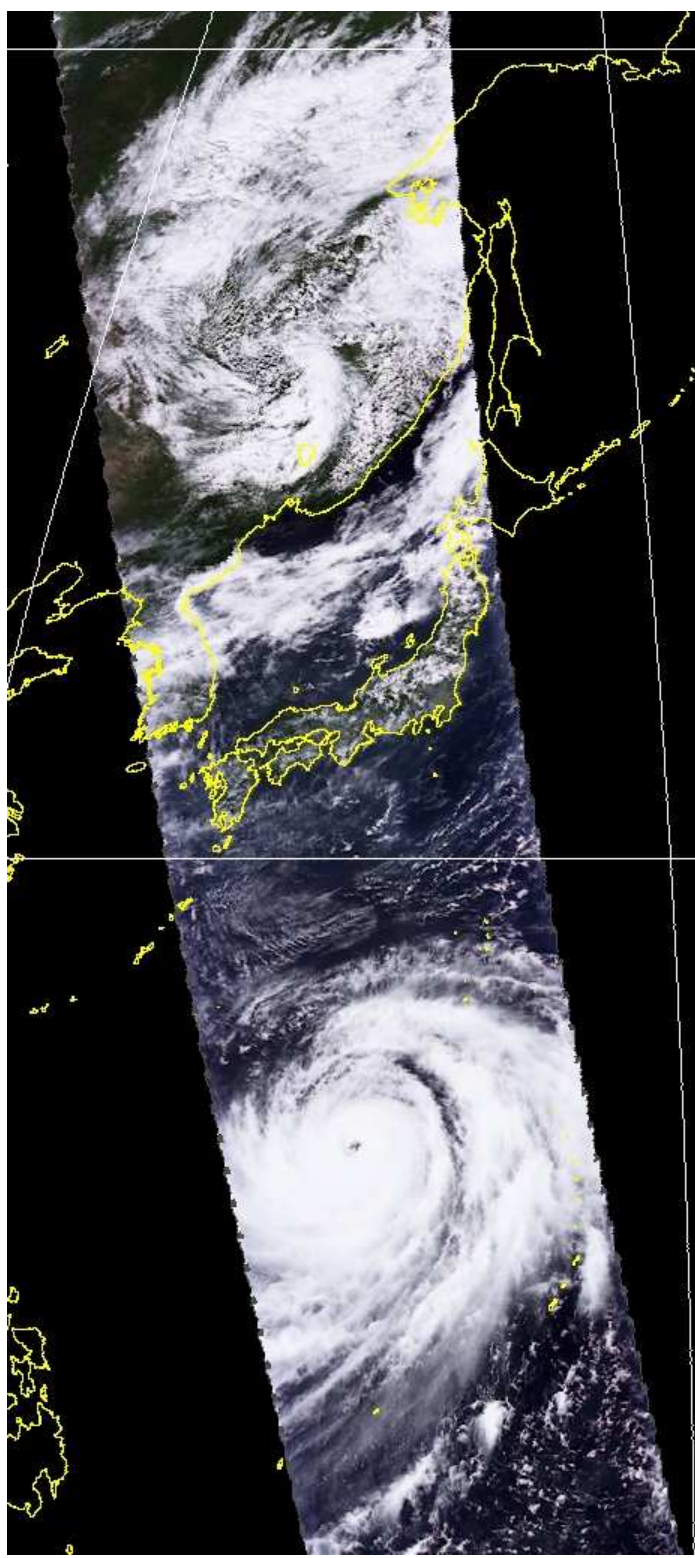

Fig. 1. POLDER true color composite of the common scene observed by MODIS and PARASOL on 2 September 2005 (MODIS swath is larger than POLDER but only the common part is presented here). The southern part of the image is typhoon Nabi as it moved North toward Japan.

for determination of optical thickness, effective particle radius and ice/liquid water content.

Recent efforts have been made to derive cloud thermodynamic phase from satellite-based passive radiometric observations (e.g., Hutchison et al., 1997; Knap et al., 2002; Baum et al., 2000; Key and Intrieri, 2000; Goloub et al., 2000; Platnick et al., 2003) but no single method is expected to be preferable for all cloud types and regions. Atmospheric or surface properties can bias existing methods or lead to ambiguous results. Further, a single unambiguous answer is inappropriate for multilayer cloud systems (Baum et al., 2003; Pavolonis and Heidinger, 2004) or mixed phase clouds (Pavolonis et al., 2005). Yet, both cases are recognized as significant components of the global cloud cover (Hahn et al., 1982, 1984; Tian and Curry, 1989). There is a great interest for new approaches that can provide more meaningful cloud thermodynamic phase information from passive imagers on a global scale.

The approach proposed in this study is based on the synergy between the POLDER-3/Parasol (POLarization and Directionality of the Earth Reflectances) and MODIS/Aqua (MODerate resolution Imaging Spectroradiometer) instruments operating in the framework of the A-Train mission. Both POLDER and MODIS have been used to derive key parameters needed to improve our knowledge of cloud properties (Platnick et al., 2003; Parol et al., 2004).

The potential of using polarization measurements of the reflected shortwave radiation to infer cloud phase has been clearly demonstrated using POLDER observations (Goloub et al., 2000; Riedi et al., 2001). The MODIS instrument provides information on cloud phase using two methods that rely on spectral measurements in the visible, shortwave to midrange infrared, and thermal infrared (Platnick et al., 2003). However, both the instruments and the specifically designed retrieval algorithms have limitations that need to be understood and recognized to prevent drawing misleading conclusions from analysis of the data products. Fortunately, limitations from one instrument can partly be mitigated by capabilities available from the other, as will be demonstrated in this paper.

We present a methodology to combine these three different and well documented approaches for deriving cloud phase within a single algorithm that uses data from both the MODIS and POLDER instruments. A brief description of the processing algorithm used to merge radiance data from MODIS and POLDER is provided in Sect. 3.

POLDER level 1b (L1B) data files correspond to a complete daytime portion of an orbit (equivalent to approximately $40 \mathrm{~min}$ of data acquisition). For each POLDER L1B file, the corresponding MODIS data (radiances and geolocation) are collocated with POLDER data on a common sinusoidal grid centered on the POLDER ascending node longitude. For each element within that grid $(6 \mathrm{~km} \times 6 \mathrm{~km})$ observed by both instruments, we form a data structure that contains all multidirectional and multispectral POLDER data together with all multispectral and multiresolution MODIS data. For MODIS, within each element of the grid, the mean and standard deviation for each radiance field is computed and kept along with all individual full resolution data $(250 \mathrm{~m}$, $500 \mathrm{~m}$ and $1 \mathrm{~km}$ ). These L1B radiances are provided together with surface albedo information derived from MODIS (Moody et al., 2005) and meteorological data (obtained for the European Center for Medium-Range Weather Forecast) needed for retrieval of cloud properties. 
In the first part of the paper, we provide an overview of the theoretical basis of the three methods for cloud phase discrimination with particular emphasis on their respective strengths and limitations. In the second part, the processing scheme for the joint MODIS/POLDER data analysis is briefly discussed and the practical implementation of the joint algorithm described. A case study, featuring typhoon Nabi on September 2005, is then analyzed to show how this synergistic algorithm can be used routinely to derive a cloud phase index containing information on retrieval quality/confidence useful for easily discriminating ambiguous from confident cases.

The resulting product is provided as a semi-continuous index ranging from confident liquid only to confident ice only instead of the usual liquid/ice/mixed discrete phase classification. This approach is a necessary step towards being able to assess errors inherent in derived regional and global cloud products.

Our results will have future implications in two domains. First, the development of the merged POLDER/MODIS phase product aims at creating a reference dataset that can be used for model evaluation or other cloud climatologies assessment. Also, the subset of high confidence cases in our dataset can be used to study liquid/ice phase transition as a function of other thermodynamics parameters with minimum bias due to temperature dependency of the phase detection technique itself. This is critically needed to understand phase transition in the supercooled temperature range where both ice and liquid can coexist. Second, the present study provides a framework for algorithm development of the upcoming GLORY mission and can be used to define mission requirements for development of future missions that would include multispectral, multiangle and multipolarization measurements.

\section{Theoretical basis overview}

Figure 2 provides an overview of the three metrics used for phase discrimination in this study. The interpretation of these metrics will be discussed. For a case study to illustrate the theoretical basis for the three different methods, we have selected a region covered by Typhoon Nabi.

\subsection{Multiangle polarization measurements}

When considering a cloudy system observed from a satellite, the polarized component of the upwelling radiance comes primarily from the upper portion of the cloud layer. Calculations have shown that the polarized component, $L p$, is saturated for cloud optical thickness greater than 2-3 depending on the cloud microphysics that are represented by the particle shape and effective size (Goloub et al., 2000; Chepfer et al., 2001). The important quantity for determining cloud phase is the polarized radiance $L p$, which is less sensitive to mul-

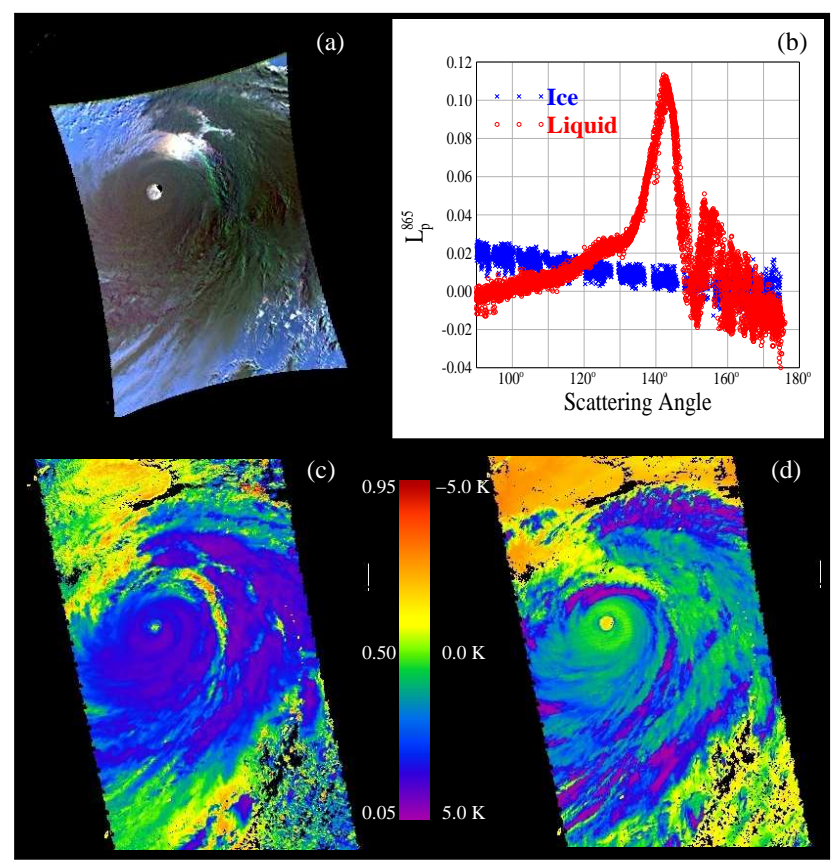

Fig. 2. Illustration over typhoon Nabi of the 3 metrics used for phase determination: (a) false color composite from 490, 670 and $865 \mathrm{~nm}$ polarized reflectance for one instantaneous POLDER field of view, (b) typical multi-angular polarized reflectance signature (from POLDER) of liquid (red) and ice (blue) clouds, (c) ratio of shortwave infrared to visible MODIS channel (2.1 to 0.865 micrometer), (d) brightness temperature difference between 8.5 and $11 \mathrm{mi}-$ crometer MODIS channels.

tiple scattering effects than the total radiance $L^{1}$. Thus, the polarization features, which correspond to single scattering, are preserved in $L p$.

According to both theory and observations (Chepfer et al., 2001; Bréon and Goloub, 1998; Goloub et al., 2000), the polarization features of clouds depend strongly on the particle shape and size. Within the range of scattering angles that can be observed by POLDER, clouds composed of liquid spherical particles exhibit a strong maximum in $L p$ at about $140^{\circ}$ (primary rainbow). Liquid clouds also exhibit a polarization value of zero (i.e., a neutral point) at around $90^{\circ}$, and supernumerary bows for angles greater than $145^{\circ}$. On the other hand, clouds composed of non spherical particles (such as ice crystals of various habits) produce a moderate positive polarization that decreases as the scattering angle increases (Fig. 2b). These different features make possible the discrimination between clouds composed of spherical (liquid) and non-spherical (ice) particles. For the present study the following criteria are used to identify liquid phase: (i) presence of a strong peak around $140^{\circ}$ using a simple threshold technique, (ii) increasing polarization with scattering angle

\footnotetext{
${ }^{1}$ Also, the polarization degree, defined as the ratio of $L p$ over $L$, is subject to multiple scattering effects since it depends on $L$.
} 
before $135^{\circ}$, (iii) negative polarized reflectance before $100^{\circ}$. For ice clouds detection we look for: (i) the absence of the cloudbow around $140^{\circ}$ using a simple threshold technique and (ii) decreasing polarization with increasing scattering angle. The results of these individual tests are then combined using a look-up table filled from a priori knowledge and yields one of the following results : confident ice, ice, unknown, liquid or confident liquid. A thorough description of the use of polarization for cloud phase determination from POLDER can be found in Goloub et al. (2000) and Riedi et al. (2001).

Because it relies on the different single scattering properties of spherical and non-spherical particles, this cloud phase detection may be considered a cloud particle shape detection method. Ice clouds are typically composed of nonspherical particles, whereas liquid water clouds are assumed to be composed of spherical droplets.

Finally, the use of polarized angular signatures depends on the availability of particular scattering angles, which means phase information will depend on the total number of viewing geometries and the range of scattering angles available. An advantage of this technique is that it is insensitive to particle size and also to potential biases in inferred cloud temperature. A complete description of the operational implementation for this algorithm is given by Riedi (2001).

\subsection{Shortwave infrared and visible measurements}

Pilewskie and Twomey (1987) recognized that reflectance measurements near 1.6 and $2.1 \mu \mathrm{m}$ can provide useful information for cloud phase discrimination. For wavelengths in the shortwave infrared spectrum, the imaginary part of the refractive index becomes non-negligible for both water and ice, thereby causing absorption by cloud particles to occur at these wavelengths. For the MODIS 1.6 and $2.1 \mu \mathrm{m}$ bands, the imaginary part is also greater for ice than for liquid water. With all other properties being equal, an ice cloud will exhibit higher absorption than a liquid cloud.

Since almost no absorption occurs at visible wavelengths, the ratio of shortwave infrared (SWIR) to visible (VIS) reflectances will be lower for an ice cloud than for a liquid cloud, assuming everything but phase is kept the same. Because ice particles are generally larger than liquid cloud droplets, we can derive cloud phase by simply applying threshold tests on the SWIR to VIS ratio. This will work as long as (i) the liquid cloud droplets are small or ice crystals large enough (see Sect. 4 for details on threshold values), (ii) the optical thickness is sufficiently large for the absorption signal to build (for very thin clouds almost no absorption occurs, hence the SWIR/VIS ratio stays close to unity for both ice and liquid clouds), and (iii) the surface albedo in the SWIR band relative to the visible is not too different.

With these considerations, the ratio of SWIR/VIS reflectances can provide useful information on the cloud thermodynamic phase when the optical thickness is greater than about 1 (King et al., 2004) and as long as the cloud is not composed of internally mixed ice and liquid particles (Lee et al., 2006). As illustrated in the following section, an obvious limitation of this metric is that separation between ice and liquid phase can be ambiguous when the particle size becomes too large for liquid clouds or too small for ice clouds. However, this technique is not subject to cloud temperature bias nor systematic geometrical sampling effects except for the sunglint region over ocean.

\subsection{Use of thermal infrared measurements}

The bispectral technique discussed in Baum et al. (2003) and Platnick et al. (2003) is currently used for routine analysis of MODIS data. It is based on the fact that the imaginary part of the refractive index for ice and liquid are almost equal at $8.5 \mu \mathrm{m}$ but diverge significantly around $11 \mu \mathrm{m}$ with ice having greater absorption. The use of differential absorption properties is similar to the previous SWIR/VIS technique but thermal infrared (TIR) channels are used, making the inference of cloud phase independent of solar illumination. With this method, cloud analyses will be consistent between daytime and nighttime conditions.

Radiative transfer simulations indicate that the brightness temperature difference between the 8.5- and $11-\mu \mathrm{m}$ bands (hereafter denoted as BTD[8.5-11]) tends to be positive in sign for ice clouds that have a visible optical thickness greater than approximately 0.5 . Water clouds of relatively high optical thickness tend to exhibit negative BTD[8.5-11] values of generally less than $-2 \mathrm{~K}$.

In addition to the differences caused by the divergence in the refractive index for ice and water, the BTD[8.5-11] values are quite sensitive to atmospheric absorption, especially by water vapor. Also, these IR window bands are sensitive to the surface emittance properties. Recent studies have shown that the $8.5-\mu \mathrm{m}$ surface emittance can be much lower than that at $11-\mu \mathrm{m}$ over non-vegetated land (Seemann et al., 2008). Thus, clear-sky BTD[8.5-11] values tend to be negative because of the difference in surface emissivity over nonvegetated surfaces between the two bands as well as from differing sensitivities to water vapor absorption. The BTD[8.511] value for low-level water clouds tends to become more negative as the water vapor loading increases and as particle size increases. Additionaly, small particles tend to increase the BTD[8.5-11] values relative to large particles (Takano et al., 1992) because of increased scattering (assuming total ice water content is kept constant).

In summary this technique is subject to surface emissivity, water vapor, and to a lesser extent particle size biases but has the major advantage of being applicable to both daytime and nightime measurements. There are known problems with this approach, primarily for optically thin cirrus, multilayer cloud systems in which optically thin cirrus overlies a low-level water cloud, and a single-layer cloud at temperatures between $238 \mathrm{~K}$ and $273 \mathrm{~K}$. In this temperature range, 
one could make a case on the basis of radiative transfer simulations that either water or ice particles could be present (or a mixture of the two), so that one cannot make an unambiguous assessment of the phase. This is important because singlelayered clouds of wide spatial extent having cloud-top temperatures in the range between $238 \mathrm{~K}$ and $270 \mathrm{~K}$ are prevalent in the storm tracks in both the Northern and Southern Hemispheres. Despite these limitations, the MODIS bispectral IR algorithm reports an unambiguous cloud phase determination in approximately $80 \%$ of global data.

The MODIS operational algorithm provides two sets of information for cloud phase. One product is provided day and night at $5-\mathrm{km}$ resolution and is based solely on the bispectral thermal infrared technique. The other product, used in the optical properties retrieval, is provided at $1-\mathrm{km}$ resolution during the daytime only and is based on a combination of both SWIR, thermal IR, and additional information from individual cloud mask tests. However, the synergistic algorithm developed in this study uses the MODIS 1-km radiances averaged over a POLDER pixel at a constant resolution of about $6 \mathrm{~km} \times 6 \mathrm{~km}$ for both the SWIR and TIR channels.

\section{Algorithm description}

\subsection{Data fusion}

The rationale for merging the three methods previously described is twofold. First, because each method has its own limitations, it is not always possible to provide a definitive phase determination based on a single technique. By implementing multiple approaches, the phase information content can be improved.

The second reason is that when all three methods provide a "reliable" answer, a general agreement between them provides a higher confidence level in the retrieval. When they disagree, this information is again useful because it provides guidance for focused attention and potential for identification of multilayer situations or mixed phase clouds.

\subsection{Implementation}

In a first step, the algorithm is designed to compute a cloud phase index from each of the three individual methods above described. All algorithms are applied on POLDER and MODIS measurements at a spatial resolution of $6 \mathrm{~km} \times 6 \mathrm{~km}$ corresponding to POLDER level 1 full resolution data with MODIS radiances being collocated and averaged over each corresponding POLDER pixel).

In our implementation, the polarization (POLDER) and bispectral thermal IR (MODIS) algorithms are obtained directly from application of a copy of the most recent production code software. However, we allow for more undetermined cases in the POLDER algorithm output when the decision is known to be less reliable. This is the case when the rainbow region (around $140^{\circ}$ ) is not observed, when the accessible range of scattering angle is lower than $10^{\circ}$ or if less than 4 viewing geometries are available. Additionally, the cloud top pressure sanity check is not performed. Further details are provided in the Algorithm Theoretical Basis Documents for each method and their associated publications (Platnick et al., 2003; Riedi, 2001).

The SWIR/VIS method does not reproduce the more complex algorithm used in the MODIS optical properties phase product (Platnick et al., 2003) (In MOD06 Collection 5 product: refer to Science Data Set Cloud_Phase_Optical_Properties). Only SWIR/VIS thresholds are applied ( $2.1 \mu \mathrm{m}$ to $0.670 \mu \mathrm{m}$ ratio over land; $2.1 \mu \mathrm{m}$ to $0.865 \mu \mathrm{m}$ ratio over ocean) and are given thresholds that provide an unambiguous decision of either ice or liquid phase. Such an implementation of the SWIR/VIS phase algorithm alone would yield a large proportion of undetermined cases if it was to be applied as a standalone phase retrieval scheme. This is counterbalanced here by the combination of the three methods.

After each method has been applied, we obtain for each pixel a triplet of index of the form [phase polar $_{\text {; }}$ phase SWIR $_{\text {in }}$ phase $\mathrm{IR}$, where phase $_{\mathrm{xxx}}$ can be either confident liquid, liquid, mixed, ice, confident ice or unknown (Fig. 3). Note that due to the inherent potential temperature bias of the IR technique, the phase ${ }_{\text {IR }}$ index is never attributed the "confident" index. In a subsequent step, a global phase index is derived from these three individual indices based on a logical decision tree presented in Fig. 4.

The decision tree starts by looking at the thermal IR phase index. The main reasons for this are that (i) the algorithm can be applied everywhere with a minimum amount of undetermined cases and (ii) it can be applied regardless of solar illumination. The use of this infrared method as a basis for the decision logic has the advantage of providing a maximum coverage of the final phase product and is expected to provide better day/night continuity.

Then, for each possible output of the thermal IR method (liquid, mixed, ice, unknown) the two other indices (phase $_{\text {polar }}$; phase SWIR $_{\text {) }}$ are compared and a value is attributed to phase $e_{\text {final }}$ depending on whether the two indices are in agreement with each other, and also whether they agree with the thermal IR test. The confidence level of each individual index is also considered for this decision. At this

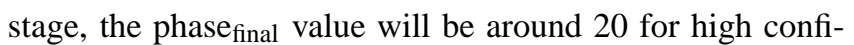
dence liquid, 50 for confident liquid, 80 for low confidence liquid, 100 for mixed cases, 120 for low confidence ice, 150 for confident ice and 180 for high confidence ice with the exact value depending on the precise triplet combination.

Finally, the exact value of the phase final $_{\text {index is set ac- }}$ counting for the potential strengths and weaknesses of each method as summarized in Table 1. This last step (called

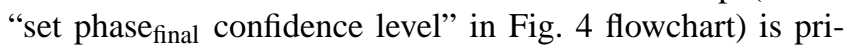
marily intended at identifying the different triplet combinations using specific values and refine the broad classification 


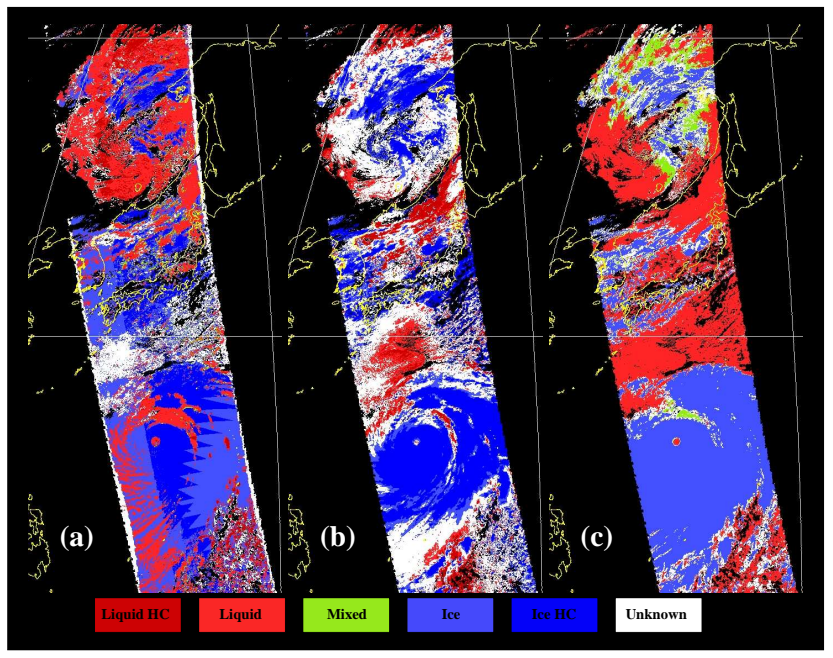

Fig. 3. Results of the partial cloud phase index retrieved from (a) POLDER polarization algorithm, (b) MODIS SWIR based algorithm and (c) MODIS bispectral IR algorithm.

(ice/liquid with high confidence, medium confidence, low confidence and mixed) made in previous step.

Each possible combination of the [phase polar $_{\text {; }}$ phase $_{S w I R}$; phase $\left._{\mathrm{IR}}\right]$ triplet is evaluated according to this general logic and accorded a value ranging from 0 (corresponding to high confidence liquid) to 200 (for high confidence ice). The highest confidence ( 0 or 200) is reached when all three indices agree with each other and are all at the highest possible confidence level for the class considered (the term class refers here to either the liquid, mixed or ice category). Intermediate values of the phase index gradually indicate the level of confidence in the retrieved phase. Values from 0 to 80 will indicate liquid phase from highest to lowest confidence, and values from 120 to 200 will correspond to ice clouds with increasing confidence. Values between 80 and 120 will denote mixed cases.

As an example, if two indices indicate liquid and the third index is ice, the final decision would be liquid but with a low confidence value (about 70). When one or two tests can not provide useful information, the remaining test will be used alone to take a decision and will always be accorded a low confidence value. Note that the final decision is not strictly a linear average of the three intermediate results as each combination has been evaluated individually. Hence, a combination of 2 liquid and 1 ice intermediate results will not always provide the same final decision depending on which methods yielded the ice answer.

The mixed phase situations tend to be denoted by a final index value around 100 and correspond to cases in which the individual tests do not agree with each other. It is important to note that "mixed" phase from a passive remote sensing point of view frequently corresponds to multilayer situations with different phases for each layer. These can not be sep-

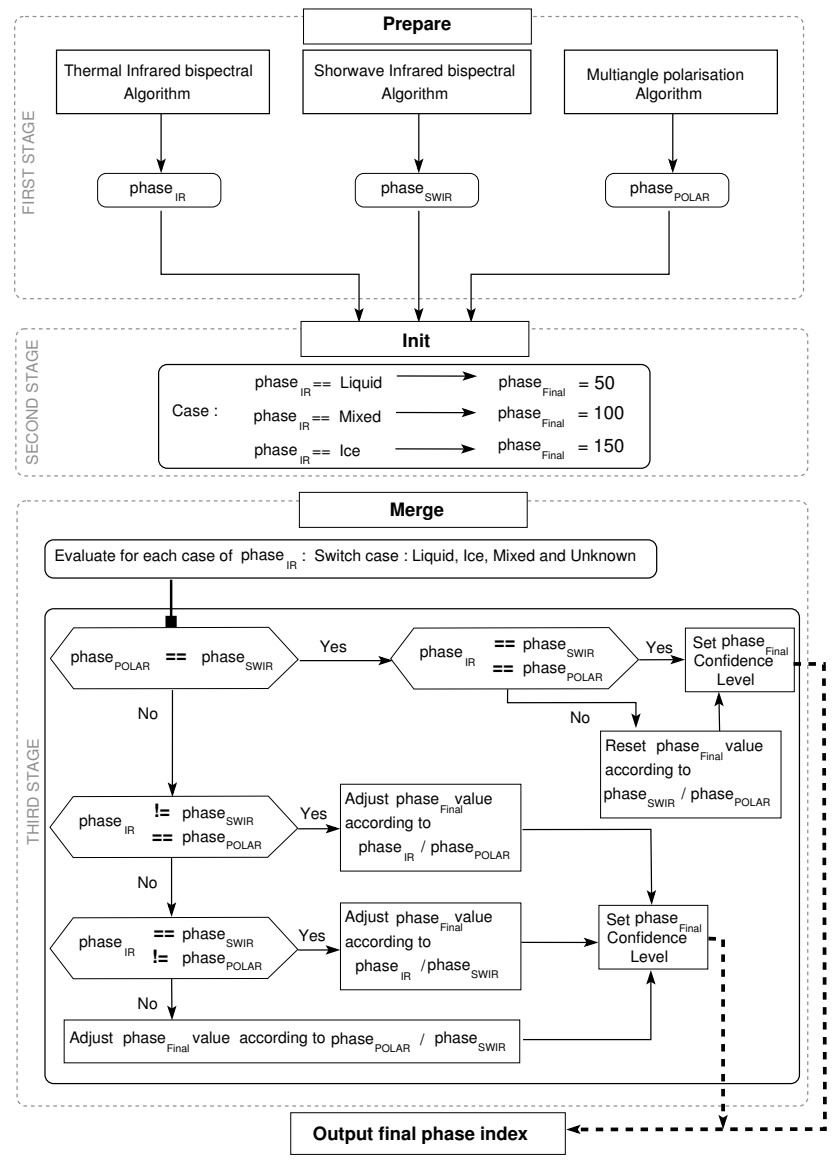

Fig. 4. Flowchart of the merging algorithm and decision tree used to produce the final cloud phase index.

arated from "real" mixed phase clouds where both ice and liquid at present at the same time. For instance a thin cirrus over a liquid cloud or a supercooled layer of liquid on top of an ice cloud may not be distinguishable from an "internally" mixed phase cloud. It is expected however that further evaluation of our merged phase product in view of CALIPSO and CloudSat observations will help understanding and discriminate these different situations.

A typical mixed phase case situation would be for instance, a thermal IR index of mixed, a SWIR indicating ice and a polarization index indicating liquid. Hence, there is a difference in the final product between low confidence ice or water and mixed phase although the final index over an image has an almost continuous range of values from pure liquid to pure ice.

Finally, when none of the three algorithms is able to provide an indication of cloud phase (ie. all three initial indices are Unknown), the final phase index will remain Unknown and is therefore distinguishable from Mixed cases which usually indicate inconsistent detection among the initial three indices. 
Table 1. Summary of phase algorithm capabilities and limitation.

\begin{tabular}{|c|c|c|c|}
\hline & Polar. & SWIR/VIS & Thermal IR \\
\hline & \multicolumn{3}{|c|}{ Sensitivity To Cloud Properties } \\
\hline Particle shape & yes & no & no \\
\hline Particle absorption & no & yes & yes \\
\hline \multirow[t]{2}{*}{ Particle size } & no & yes & moderate \\
\hline & \multicolumn{3}{|c|}{ Potential Biases } \\
\hline Obs. Geometry & yes & no & no \\
\hline Temperature profile & no & no & yes \\
\hline Water Vapor profile & no & no & yes \\
\hline Particle Size & no & yes & moderate \\
\hline Fractional Cloud Cover & yes & moderate & moderate \\
\hline Surface albedo/emissivity & no & yes & yes \\
\hline
\end{tabular}

\section{Theoretical performance consideration}

Before considering the case study, we discuss a number of theoretical scenarios to understand how the algorithm performs in particular situations. To help in understanding this theoretical discussion, we have summarized in Table 1 the basic principles and potential biases for each of the three methods used to develop the joint algorithm.

\subsection{Single-layered cirrus}

The inference of phase for optically thin ice clouds (i.e., cirrus) can be problematic because both surface spectral properties and emissivity can bias the SWIR and thermal IR methods.

For the SWIR test, the thresholds have values such that in case of thin clouds, the ratio could be in the undetermined range. One exception is in the sunglint region over ocean where the ratio can be high enough to lead to liquid phase. Over ocean in the sunglint region of MODIS, we use the POLDER data to evaluate the "out of glint" cloud reflectance. If the minimum reflectance at $0.865 \mu \mathrm{m}$ observed by POLDER out of the glint region is lower than 0.1, the SWIR test is switched off to prevent false detection of liquid phase.

The thermal IR test is very sensitive to thin cirrus due to the strong signal of the BTD[8.5-11]. However, when the cirrus optical thickness is less than approximately 0.5 over oceans and warm, vegetated surfaces (where the surface emissivity is high in both IR bands), a false inference of liquid phase can occur (Baum et al., 2003). The misinterpretation of phase can occur at slightly higher values of cirrus optical thicknesses over non-vegetated surfaces such as deserts.

Fortunately, the problem is reversed for polarization measurements since clear skies yield a similar angular signature to ice clouds or very broken low liquid clouds. Hence, by de- fault the polarization will tend to identify correctly thin cirrus clouds as ice clouds.

Overall, the thin cirrus may be partially misclassified as liquid by the SWIR and IR tests but the polarization tests will reduce this bias.

\subsection{Thick ice/liquid clouds}

For optically thick clouds, the SWIR/VIS signal is insensitive to surface spectral properties but the potential particle size bias remains. The SWIR reflectance will saturate more rapidly than the VIS reflectance as cloud optical thickness increases. Subsequently, the SWIR/VIS ratio will decrease accordingly for both ice and liquid clouds and for all particle effective sizes.

Consequently, the probability that the SWIR/VIS ratio for liquid clouds decreases below a given threshold will increase as cloud optical thickness increases. This tendency occurs regardless of particle size. In this asymptotic regime, we can determine from theoretical values of the SWIR and VIS reflectances (computed for different values of effective radius and optical thickness), that there exists an extended range of ( $\left.\tau_{\mathrm{c}}, R_{\text {eff }}\right)$ combinations where ice and liquid phase clouds are indistinguishable using a simple SWIR/VIS ratio. Figure 5 illustrates the overlap between ice and liquid phase solution spaces in a $2.1-0.865 \mu \mathrm{m}$ reflectance diagram. Reflectances here were computed using the microphysical properties and radiative transfer models used for the operational MODIS cloud optical properties retrievals (Platnick et al., 2003).

The situation may not be as dramatic as it appears in Fig. 5 because as liquid cloud optical thickness increases, the probability of having larger droplets increases. The reverse is not necessarily true for ice clouds as thick ice clouds could present both large and small particles at cloud top. It remains that the SWIR/VIS ratio metric will provide unambiguous information only for liquid clouds with $R_{\text {eff }}$ less than 10.0 micrometer or ice clouds with $R_{\text {eff }}$ greater than 30 micrometer.

As can be seen from inspection of Fig. 3, this implies that the potential exists for numerous pixels to remain unclassified using the SWIR/VIS metric. For all these cases, the additional information from the IR bispectral and polarization techniques will be necessary to provide reliable phase determination.

The BTD[8.5-11] signal is affected by smaller particle sizes because of the increased scattering, but the information provided becomes ambiguous in case of supercooled liquid clouds when cloud top temperature is in the $238 \mathrm{~K}-270 \mathrm{~K}$ range. As with the SWIR test, one could make a case for either water or ice in this range based on a range of simulated conditions. A significant number of undetermined or mixed cases for these clouds would benefit from additional information from SWIR/VIS and polarization tests.

Note finally that for optically thick clouds, the polarization signal is strong and unambiguous as long as cloud cover 

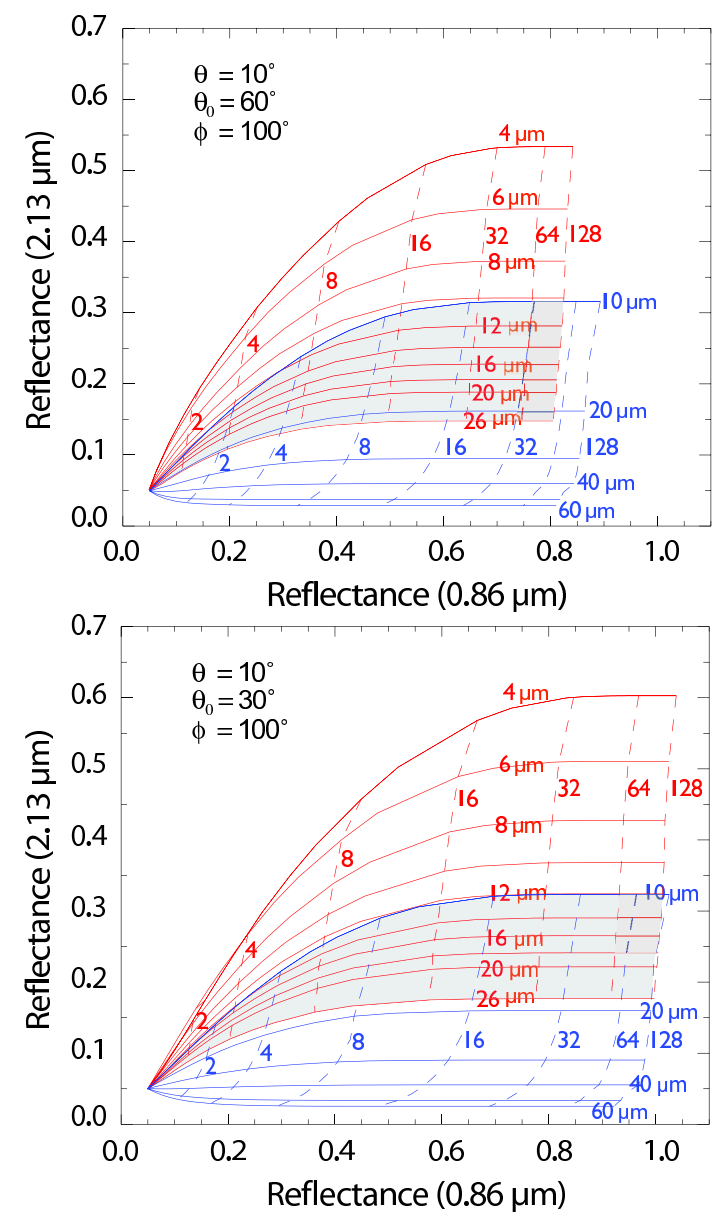

Fig. 5. Simulation for two solar and viewing geometries of the $2.1 \mu \mathrm{m}$ against $865 \mathrm{~nm}$ reflectances for an ice (blue) and liquid (red) cloud of varying optical thickness and effective radius. Overlapping zone between the ice and liquid retrieval spaces (shaded region) corresponds to situation where a SWIR vs. VIS/NIR reflectance metric can not provide unambiguous phase information.

is not broken and the angular range available is sufficient. The main limitation remaining is that polarization provides information for the top of the cloud and will not be sensitive to anything below an optical depth of 3 .

\subsection{Thin ice cloud over liquid water cloud}

Multilayered clouds, and in particular the case where an ice cloud overlies a lower-level liquid cloud, are problematic for any passive retrieval of cloud properties because for practical purposes, current operational algorithms have to assume a single-layered cloud of homogeneous phase. For multilayered clouds, a single phase obviously cannot represent the situation. As a consequence, an obvious problem occurs when a cloud optical property model has to be chosen from a static look-up table for the subsequent retrieval of optical thickness and particle effective size.

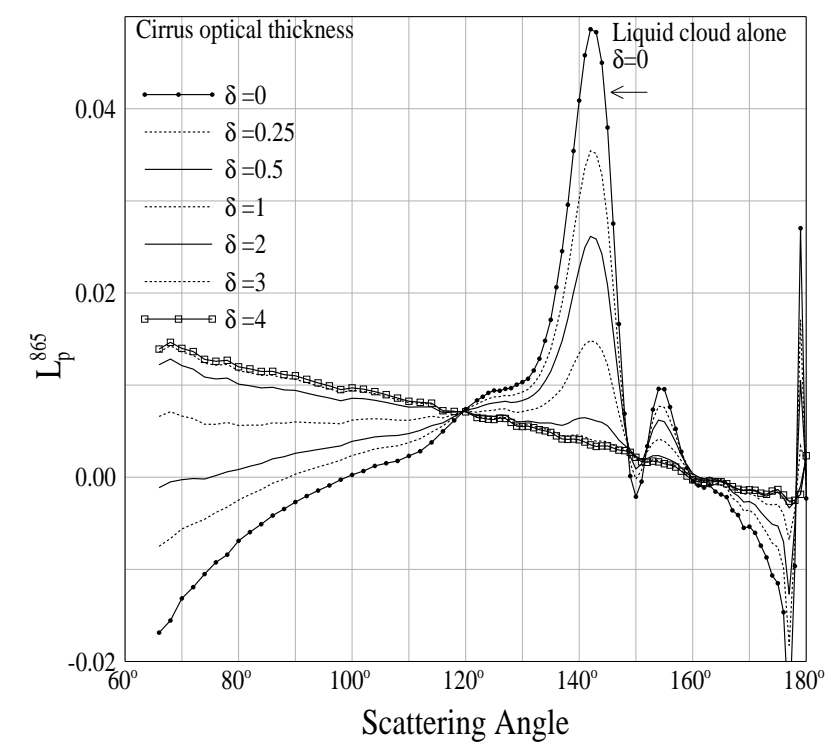

Fig. 6. Simulation of polarized reflectance at $865 \mathrm{~nm}$ as a function of scattering angle for an ice cloud of varying optical thickness overlaying a lower liquid water cloud of optical thickness 10 .

In the case of relatively thin cirrus, the different sensitivities of the three phase discrimination methods can provide useful information depending on the cirrus optical thickness. To evaluate our ability to detect a multilayered cloud situation, we have simulated the different metrics used in our algorithm for an ice cloud of varying optical thickness (between 0 and 10) overlying a lower-level liquid cloud layer of constant optical thickness (10). Figures 6, 7, 8 and 9 present respectively the simulated metrics used for polarization, shortwave infrared and thermal infrared based retrievals.

For SWIR/VIS and IR simulations, the ice crystal model is a perfect hexagonal column with an aspect ratio of 1 (length over half-width of the column, see Yang and Liou (2006) for instance). The crystal size corresponds to an equivalentvolume sphere of $40 \mu \mathrm{m}$ radius. We choose a simple model for which all optical properties can be easily and coherently computed for the various wavelengths involved in our retrievals. Though more realistic and detailed models could be use (Baum et al., 2003), we kept here a simple ice crystal model to focus on the impact of the cirrus optical thickness rather than the detailed cloud microphysics. For polarization simulations, we used the IHM model from Labonnote et al. (2001) which proved to best match the polarized angular signature of ice clouds on a global scale (Baran and Labonnote, 2006). The liquid cloud model is a gamma size distribution of spheres with an effective radius of $12 \mu \mathrm{m}$ and an effective variance of 0.1 . 
An adding-doubling code was used to compute polarized reflectances (De Haan et al., 1987) whereas an accurate Fast Discrete Ordinate Method (Dubuisson et al., 1996, 2005), which accounts for absorption and scattering, has been used for cloud radiance computations in all visible, shortwave infrared and thermal infrared bands.

Polarized reflectances tend to saturate for optical thicknesses greater than 2. For cirrus optical thicknesses less than 2 , the liquid cloud signature is still present in the polarized reflectances in the rainbow scattering angle region (around $140^{\circ}$ ) as can be seen from Fig. 6.

For a cirrus optical thickness less than 1, the polarization signature will be classified as "liquid" by the POLDER standalone algorithm. Between 1 and 2, the polarization signature will be interpreted as a "mixed" or "undetermined" case; an optical thickness greater than 2 will yield "ice" phase. If the rainbow region is not sampled by the measurements, the "mixed" case can not be identified.

The situation is more complex with the SWIR/VIS metric since the respective contribution from absorption and scattering will build up differently depending on the particle size of both the liquid and ice clouds. However, we can still evaluate approximate upper and lower limits of this metric by looking at an asymptotic regime of this ratio. Simulations are performed for two solar zenith angles (nadir and $30^{\circ}$ ), 10 view zenith and 20 relative azimuth angles.

On Figs. 7a and b, the SWIR/VIS theoretical ratios are plotted for each cirrus optical thickness, as a function of viewing angle by averaging over relative azimuth angle. This was done to provide a crude estimate for the magnitude of variation one could expect from a set of realistic cloud conditions. If we consider the average ratio for pure liquid cloud $(0.75$ for cirrus optical thickness $=0$. $)$ and allow a $0.1(0.2)$ variation from this initial value, then we can see that when thin cirrus $\tau$ is less than $0.2(0.5)$, the phase will be returned as liquid. Conversely, considering the thick ice cloud limit $(0.15)$ and allowing an equivalent $0.1(0.2)$ increase for the ratio would lead to a limit of cirrus $\tau$ of about 3.0 (2.0) above which a single-layered ice cloud can not be distinguished from a multilayered cloud scenario.

Another way to consider the problem is to consider the thresholds used for intermediate phase retrieval based on SWIR/VIS ratio. The thresholds have been set experimentally and result partially from the analysis of a large number of MODIS scenes during the validation and quality assessment phase of MODIS cloud optical properties collection 5 products. The PDF of the ratio values is divided into 5 regions using thresholds at $0.65,0.55,0.35$ and 0.25 , which correspond respectively to confident liquid, probably liquid, unknown, probably ice, and confident ice. These thresholds are demonstrated in Fig. $7 \mathrm{c}$ and d, where the SWIR/VIS theoretical ratios are plotted on a surface as a function of both cirrus $\tau$ and viewing geometry. It can be seen from this that confident liquid (ice) will be assessed only if the overlaying cirrus $\tau$ is lower (greater) than 0.2 (3.0).
The reduced confidence thresholds occur respectively for liquid and ice at cirrus optical thicknesses of about 0.5 and 2. With this single criterion, a multilayered cloud situation consisting of ice over water clouds, in which the cirrus optical thickness lies between 0.5 and 3 , will most probably lead to a low confidence or undetermined phase. Note that these threshold values, derived from statistical analysis of real observations, are consistent with those derived by allowing a 0.1 departure from pure liquid or ice theoretical ratio values. An important observation also is that this metric seems to present very limited dependance on viewing or solar geometries, which justify the applicability of fixed thresholds with respect to the observation geometry, and in consideration of other uncertainties linked to particle size, for example.

When the $11 \mu \mathrm{m}$ brightness temperature is in the range of $238 \mathrm{~K}$ to $268 \mathrm{~K}$ where ice and supercooled liquid water can coexist, the bispectral IR algorithm is basically selecting the phase based on BTD[8.5-11] values using a set of thresholds at $0.5,-0.25$ and $-1.0 \mathrm{~K}$, delimiting the regions of Ice, Unknown, Mixed and Liquid.

Figures 8 and 9 illustrate the sensitivity of BTD[8.5-11] to cirrus optical thickness and to atmospheric profile. Brightness temperatures at 8.5 and $11 \mu \mathrm{m}$ depend strongly on each cloud layer altitude (temperature), particle size, water vapor profile, and surface emissivity. It is difficult to evaluate all possible combinations of atmospheric profile, cirrus altitude and optical thickness, and liquid cloud altitude. We have chosen to illustrate only a few problems using simulations performed with four distinct scenarios.

Two cases are considered in which the liquid cloud layer is located at either $5 \mathrm{~km}$ (Fig. 8) or $2 \mathrm{~km}$ (Fig. 9), with the cirrus cloud being kept at $10 \mathrm{~km}$. For each case, two very different atmospheric profiles are considered (MidLatitude Summer, humid and warm; SubArctic Winter, dry and cold). Finally, the thresholds used for the IR phase retrieval are indicated on each of the BTD[8.5-11] figures.

A first observation is that the location of the lower liquid cloud has a moderate influence on the observed BTD[8.511], but this influence is more pronounced (as expected) for the warm/humid profile. For the MidLatitude Summer profile, the cirrus temperature is set at about $248 \mathrm{~K}$. From the corresponding BTD[8.5-11] diagrams on Figs. 8 and 9, it seems that all situations will be declared Liquid for cirrus $\tau$ up to about 2.0 and will then be declared Mixed or Unknown depending on the value of the cirrus $\tau$. This clearly illustrates the potential bias in the IR retrieval in the case of multilayered clouds in a warm/humid atmosphere. For the SubArctic Winter profile, and again for both liquid cloud altitudes, the phase will rarely be declared as Liquid since the BTD[8.5-11] value increases above the -1.0 threshold for cirrus optical thickness as low as 0.1 or 0.2 depending on the viewing geometry. The phase may be declared as Mixed for cirrus $\tau$ up to 0.5 and will be Unknown until the cirrus optical thickness reaches a value of 2 unless the $11 \mu \mathrm{m}$ BT passes below the $238 \mathrm{~K}$ threshold. 

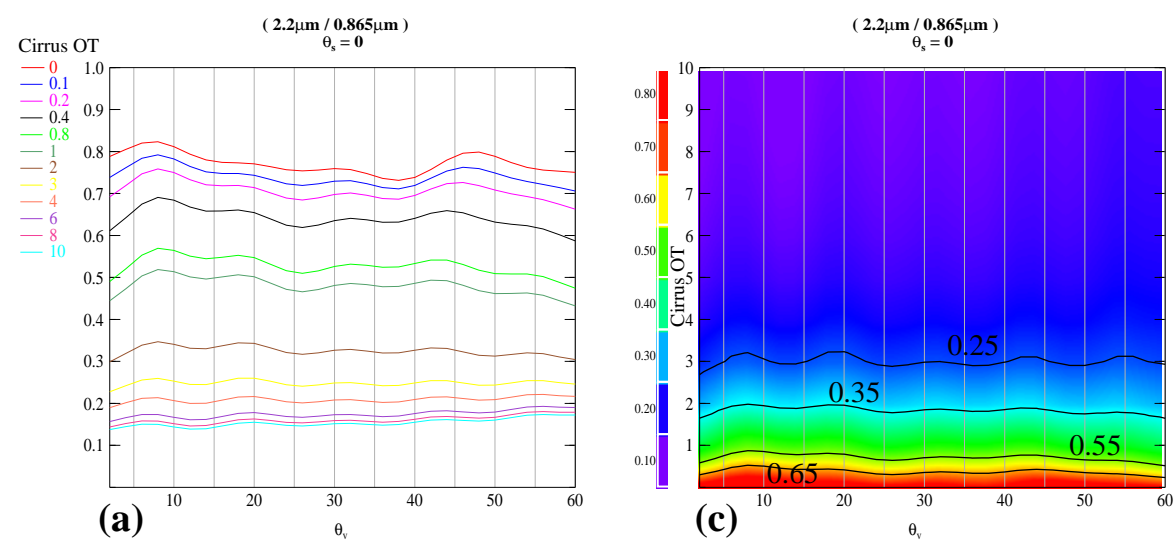

(c)
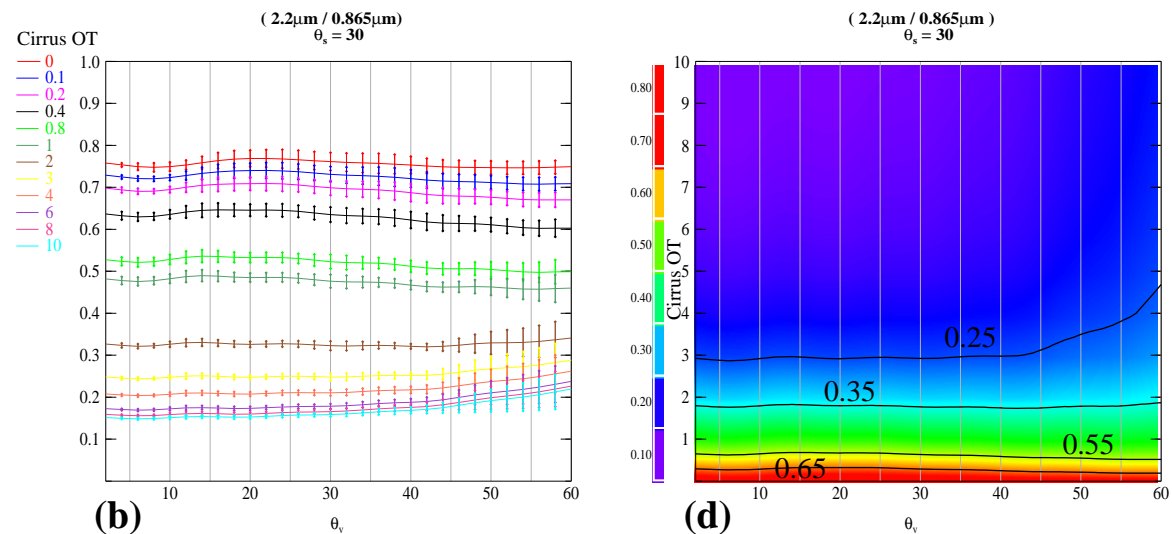

(d)

Fig. 7. Simulation for different geometries of the $2.1 \mu \mathrm{m}$ to $865 \mathrm{~nm}$ reflectance ratio for an ice cloud of varying optical thickness overlaying a lower liquid water cloud of optical thickness 10. Panels (a) and (b) present the simulated ratio for different cirrus optical thickness as a function of viewing angle. Results are averaged over relative azimuth and standard deviations are indicated using error bars. Panels (c) and (d) present the same results as a surface contour of simulated ratio, function of viewing angle and cirrus optical thickness. The latter representation is used to better illustrate the different regions defined by the SWIR vs. VIS/NIR reflectance thresholds used in our algorithm.

For additional discussion about the sensitivity of BT11 and BTD[8.5-11] we suggest reading, among others, the studies from Baum et al. (2000), Strabala et al. (1994) and Baum et al. (2003).

This sensitivity study is obviously limited but clearly illustrates again the difficulty of dealing with supercooled and/or multilayer clouds. However, we have shown that the polarization, SWIR/VIS and TIR metrics will behave quite differently in these situations, providing potential information to identify multilayer situations as shown by Nasiri and Baum (2004) using MODIS data only. The identification of multilayer situations in case of Mixed phase (as determined by our multisensors algorithm) will be done a posteriori by combining the phase index with other information such as different cloud pressure retrievals or observations from active sensors.

\subsection{Mixed phase}

Mixed phase clouds are obviously problematic and therefore of primary interest for our current investigation. When liquid spherical droplets coexist with ice particles, we anticipate that high absorption by ice will show up in the SWIR band and also that BTD[8.5-11] may indicate either mixed or undetermined phase.

However, spherical particles can produce a rainbow feature that will toggle a liquid phase detection in the polarization test. Opposing decisions from the SWIR/VIS and polarization tests, with mixed or undetermined phase from the IR test, will lead to confident mixed phase in the final index.

Again, additional information to discriminate multilayer clouds from single layer mixed-phase clouds will then be needed to decide whether mixed phase is due to vertical structure or inherent to the single cloud layer. Work is under progress to use different pressure retrievals from POLDER 


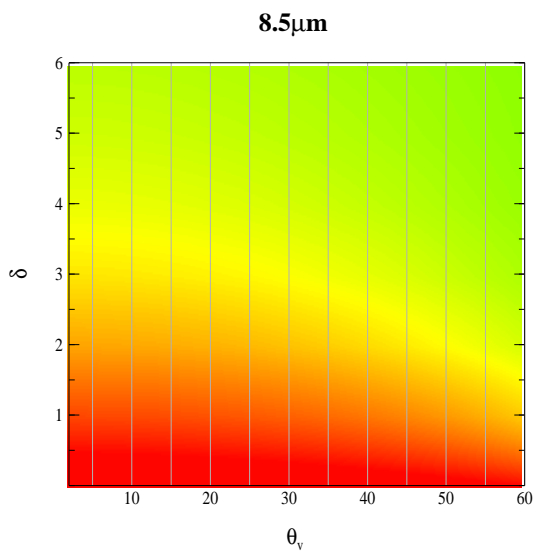

$11 \mu \mathbf{m}$
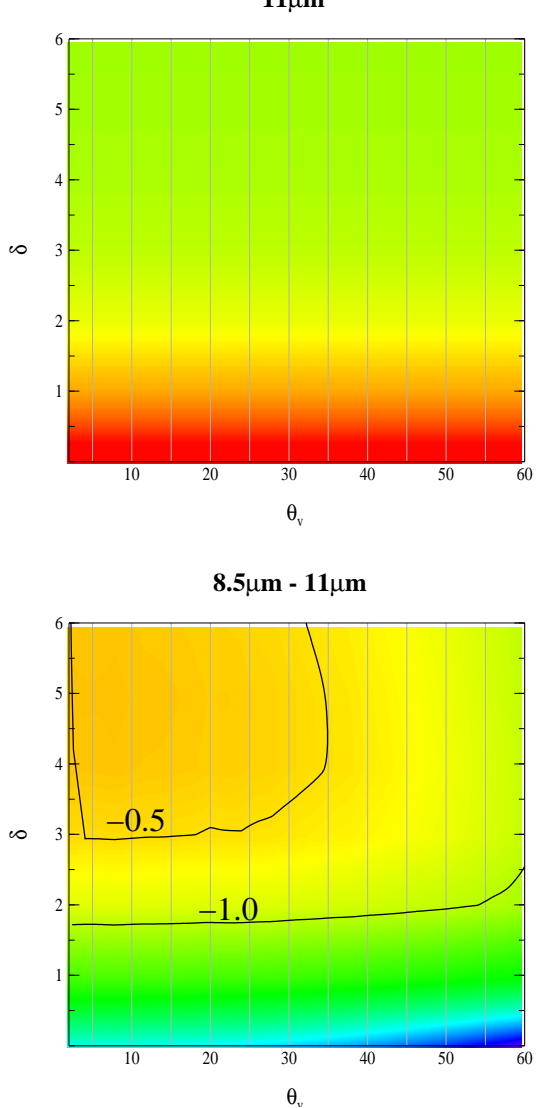

$8.5 \mu \mathrm{m}$

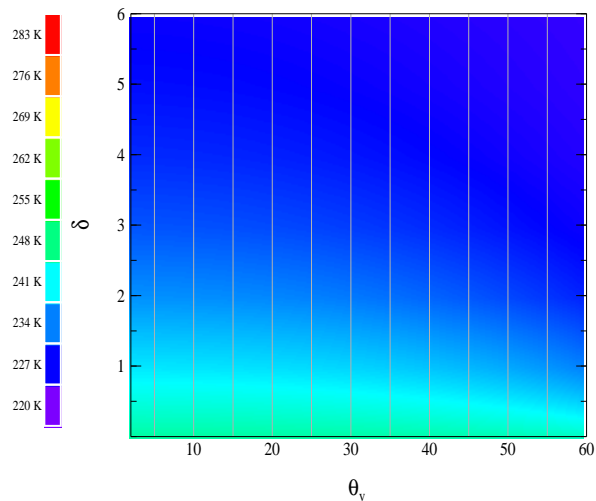

$11 \mu \mathrm{m}$
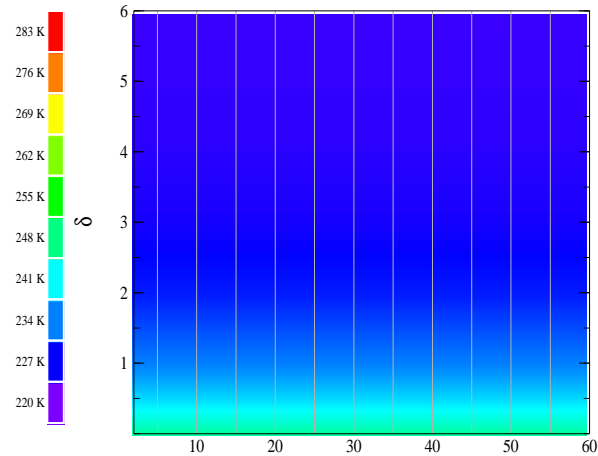

$\theta_{\mathrm{v}}$

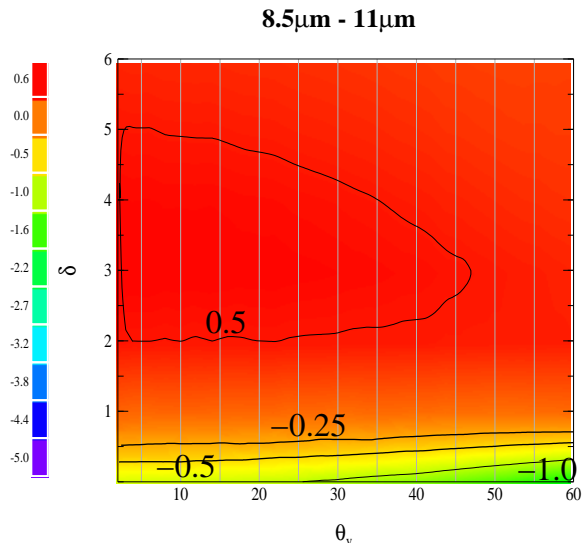

Fig. 8. Simulation for a Mid-Latitude Summer (left) and a Sub-Arctic Winter (right) atmospheric profile of the brightness temperature differences (in Kelvin) between channel at 8.5 and $11 \mu \mathrm{m}$ as a function of view angle for an ice cloud of varying optical thickness at $10 \mathrm{~km}$ overlaying a lower liquid water cloud of optical thickness 10 , located at $5 \mathrm{~km}$. Isolines correspond to the thresholds used in the bispectral IR phase determination.

and MODIS to help detect the presence of multilayer clouds. Also, the multilayer flag product available from MODIS MYD06 cloud product can provide some information and is under investigation, being a new product. Both will obviously require validation from the active lidar in the A-Train.

\subsection{Snow and sea ice}

The presence of snow on surface or sea ice is already problematic for cloud detection itself and will also impact cloud phase determination. Because the SWIR and the IR techniques rely on differential absorption between water and ice, the presence of snow or sea ice can potentially impact the 


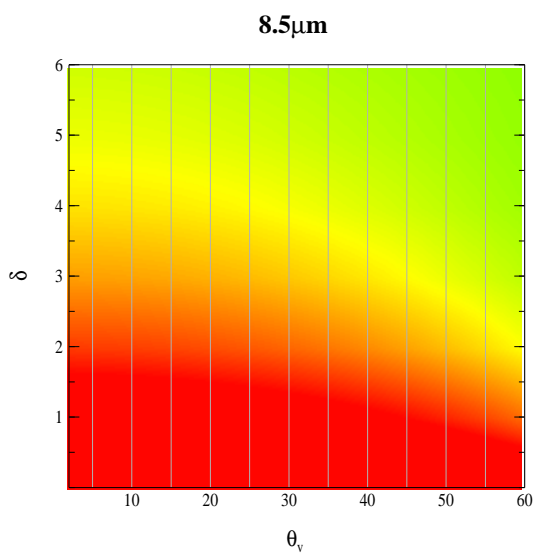

$11 \mu \mathrm{m}$

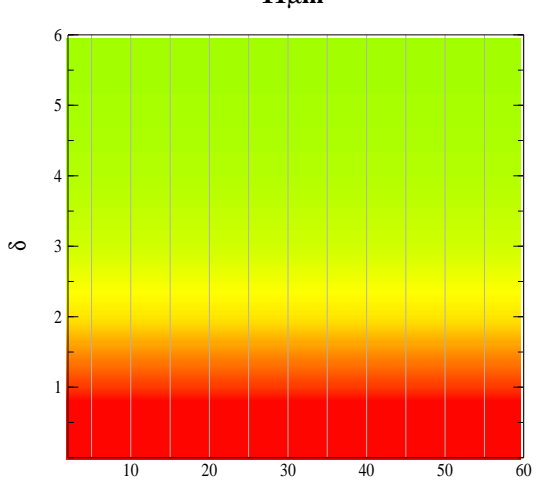

$\theta_{\mathrm{v}}$

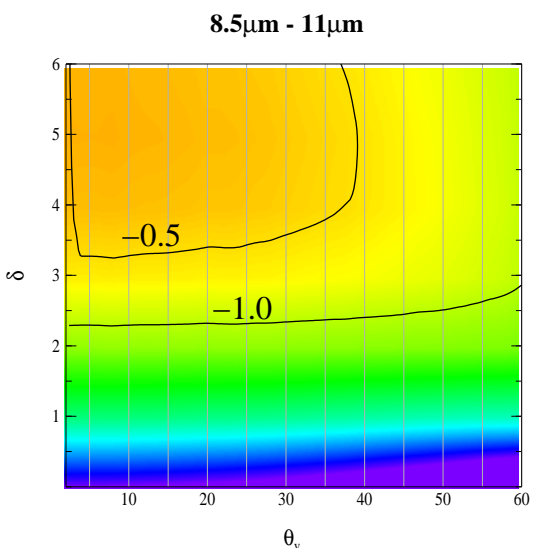

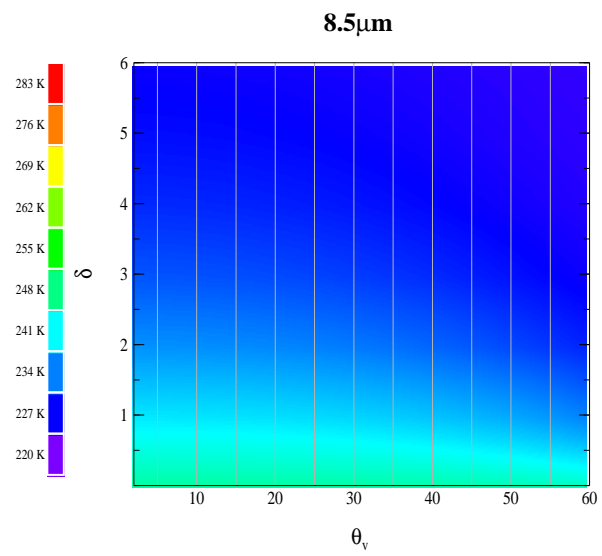

$11 \mu \mathrm{m}$
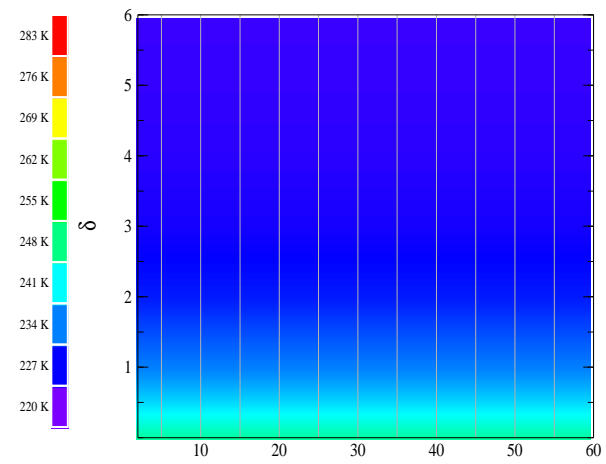

$\theta_{v}$

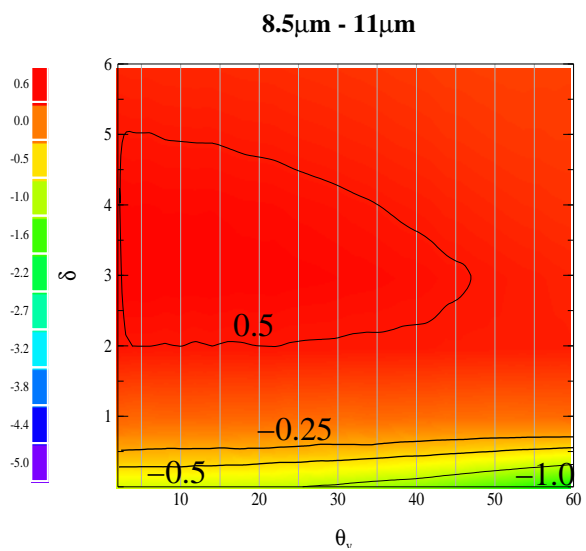

Fig. 9. Same as Fig. 8 but liquid cloud is located at $2 \mathrm{~km}$.

identification of relatively thin clouds by these two techniques.

For the SWIR technique, the situation will be mostly problematic in case of thin liquid clouds. The strong absorption by the surface tend to bias the SWIR/VIS ratio toward ice signature for all cases and results in either ambiguous or erroneous phase detection only for liquid clouds.

Regarding the bispectral IR technique, the situation is more complex as it depends on both surface temperature and temperature and water vapor profiles. However, snow sur- faces under clear sky conditions tend to present large negative values of BTD[8.5-11] and therefore would be declared as liquid by the current IR algorithm. In this case, an erroneous or ambiguous phase decision could be made for thin cirrus with optical thickness smaller than 0.5.

The polarization technique on the other hand is mostly insensitive to surface conditions (except sunglint which is highly polarized) and will not suffer from presence of snow or sea ice in the identification of cloud phase. 
Overall, the merged phase index will depend primarily on whether or not the rainbow can be observed and detected by POLDER which would be the case if observation geometry permits and cloud optical thickness is greater than 0.5.

Thin clouds with optical thickness smaller than 0.5 will therefore constitute a challenge for the phase detection if the cloud can be detected in a first place. The problem of snow/sea ice will require further analysis using CALIOP observation to evaluate thin clouds detection skills over snow/sea ice and the associated phase decisions.

\section{Case study analysis and discussion}

The case study selected to illustrate the present approach includes Typhoon Nabi on 2 September 2005 (Fig. 1). This portion of a PARASOL orbit was selected due to the presence of a typhoon over ocean and a large cloud system over land. Both scenes contain both optically thin and thick clouds at different levels, thus providing an a priori complex case for which individual phase retrieval methods might provide ambiguous information.

We discuss hereafter particular cloud situations available from this case study. For each, results for individual phase discrimination tests are discussed, as well as how each test contributes to the final decision. The final decision takes into account the limitations and advantages of each method.

\subsection{Midlevel clouds}

The cloud system in the northern part of Fig. 1 provides a very good example of a midlevel cloud layer as can been observed from the Oxygen cloud apparent pressure retrieval (Vanbauce et al., 1998, 2003) on Fig. 10b. Most of these clouds have temperatures between $238 \mathrm{~K}$ and $268 \mathrm{~K}$ where ice and supercooled liquid water can coexist. Figure 3 shows that a significant portion of the cloud system indicates an ambiguous signal for the SWIR/VIS metric that is thought to be associated with large liquid particles since both polarization and TIR tests agree on Liquid phase. The ice cloud part of the cloud system is retrieved fairly coherently in all three methods leading to high confidence ice in the final retrieval shown in Fig. 10a.

Finally, the pixels declared as Mixed phase by the TIR method are given a lower confidence Liquid flag in the polarization test and the Unknown flag from the SWIR/VIS test. As can be seen from the $\mathrm{O}_{2}$ A-band pressure retrieval, those Mixed phase pixels have a slightly higher altitude than some surrounding high confidence Ice pixels, perhaps indicating a multilayer cloud situation. This will be further evaluated using active sensors in future work.

\subsection{Typhoon scene}

Investigation of a portion of Typhoon Nabi in the southern part of Fig. 1 provides further insight as to how the three

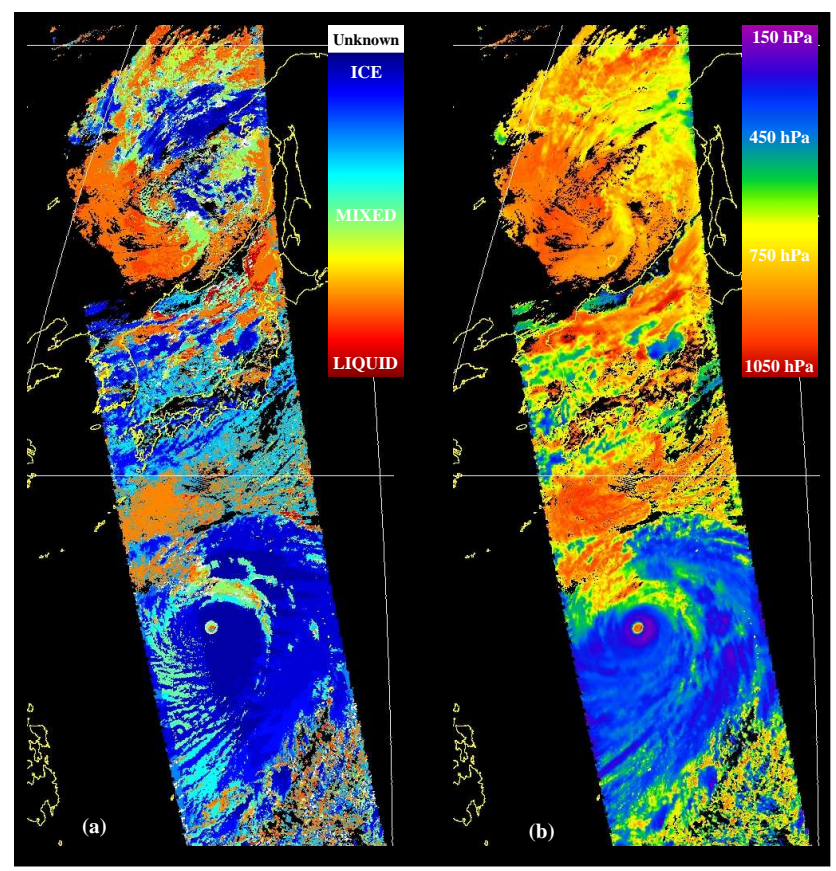

Fig. 10. (a) Results of the final cloud phase index retrieved from a combination of POLDER and MODIS data. (b) Cloud top pressure derived from POLDER oxygen A-Band method.

methods can provide a very different perspective of a given situation.

The top of the typhoon is located very high in the atmosphere and whether it is optically thick or not does not make much difference in the TIR retrieval, indicating a large extent of the cirrus cloud layer (Fig. 3c). The situation is more ambiguous again with the SWIR/VIS metric (Fig. 3b), which turns rapidly from confident Ice about the center of Nabi to Unknown without seeing very many lower confidence Ice pixels.

Finally the polarization test (Fig. 3a) indicates clearly the presence of a liquid cloud layer just north of Nabi's eye and also in the western and south-western region of the typhoon. For these pixels, the final cloud phase index range from low confidence Ice to high confidence Mixed. It is evident from inspection of individual metrics on Fig. 2 and $\mathrm{O}_{2}$ cloud pressure on Fig. 10b that most of these pixels correspond to multilayer clouds where high thin cirrus overlays a lower-level liquid cloud layer. Note also that the partial phase indices (Fig. 3) are very consistent in this particular case with what has been discussed in the theoretical part related to performance of the algoritm in case of thin cirrus over low water clouds. Again, these findings would need to be validated using active sensor observations available from CALIPSO and CloudSat in the A-Train. However, this is beyond the scope of the present paper which aims primarily at describing the theoretical basis and implementation of our synergistic algorithm. 


\subsection{Final phase index}

Figure 10a presents the final results of our joint POLDER/MODIS algorithm together with the cloud pressure derived from Oxygen A-Band (Fig. 10b). At this stage, we can comment on two facts. First, the cloud phase index presents fairly smooth variation indicating that the logical decision tree does not yield unstable situations where we would randomly switch between confident ice and confident liquid. Secondly, the phase index behaves fairly coherently compared to cloud $\mathrm{O}_{2}$ pressure even if this information is not involved in the phase retrieval process. Overall, and before a thorough validation is performed using active sensors, we conclude from this case study (and others, not shown/discussed here) that our proposed method provides new and relevant information on cloud thermodynamic phase and to a lesser extent on the vertical structure of cloud layers.

\section{Conclusions}

With the use of coincident data available from POLDER3/Parasol and MODIS/Aqua, three independent methods for deriving cloud phase have been applied singly and in combination. It is shown that these methods can provide different information for a single-layered cloud due to their respective sensitivity to different parameters. For unambiguous cases where all three methods provide the same answer individually, the combination is still useful since it can be used to assess the confidence level of the phase retrieval. For cases where the three methods disagree, an attempt is made to interpret the differences in terms of multilayer clouds and/or single-layered mixed phase clouds.

Validation of the retrieved joint product is outside the scope of this paper but we can expect the combination of well evaluated methods to provide at least an equally accurate product. The value added by the synergy of POLDER and MODIS relies mainly in (i) the semi continuous phase index which values can be interpreted in terms of confidence in the retrieval, (ii) the potential to clearly identify mixed phase cases and (iii) to a lesser extent, the possibility of determining an index for almost every pixel that uses the strengths of each method.

In future research, a thorough analysis of the full joint dataset provided by POLDER3/Parasol and MODIS/Aqua will provide statistics of this new product. Obviously, with the availability of CloudSat and CALIPSO, it is expected that a large validation dataset containing vertical profile information will help us in evaluating the statistical meaningfulness of each class of the decision look-up table.

If a reasonable correlation between radar/lidar data and the phase index can be demonstrated in case of multilayer or mixed phase clouds, the POLDER/MODIS combination will prove extremely useful to extend the vertical informa- tion from the active instruments to the full swath covered by the passive instruments of the A-Train.

Finally, this work demonstrates the interest for a sensor which could provide both multiangle, polarization and extended multispectral observations. In a near future, this method could be adapted to observations from the Glory mission before a next generation spectropolarimeter can be developed and launched.

Acknowledgements. The authors are very grateful to CNES and NASA for providing the POLDER and MODIS data. Cecile Oudard and Francois Thieuleux were supported by University of Lille, region Nord-Pas-de-Calais, CNRS and CNES, in the framework of the ICARE project. This research project was supported by CNES and the Programme National de Teledetection Spatial. Finally, the authors would like to acknowledge the two reviewers for their numerous comments and help in improving the manuscript.

Edited by: T. Garrett

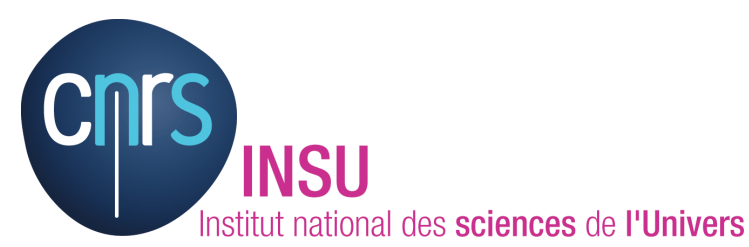

The publication of this article is financed by CNRS-INSU.

\section{References}

Baran, A. J. and C.-Labonnote, L. : On the reflection and polarization properties of ice cloud, J. Quant. Spectrosc. Ra., 100, 41-54, 2006.

Baum, B. A., Soulen, P. F., Strabala, K. I., King, M. D., Ackerman, S. A., Menzel, W. P., and Yang, P.: Remote sensing of cloud properties using MODIS airborne simulator imagery during SUCCESS: 2. Cloud thermodynamic phase, J. Geophys. Res., 105, 11781-11792, 2000.

Baum, B. A., Frey, R. A., Mace, G. G., Harkey, M. K., and Yang, P.: Nighttime multilayered cloud detection using MODIS and ARM data, J. Appl. Meteor., 42, 905-919, 2003.

Baum, B. A., Heymsfield, A. J., Yang, P., and Bedka, S. T.: Bulk scattering models for the remote sensing of ice clouds. Part 1: Microphysical data and models, J. Appl. Meteor., 44, 18851895, 2005a.

Baum, B. A., Yang, P., Heymsfield, A. J., Platnick, S., King, M. D., Hu, Y.-X., and Bedka, S. T.: Bulk scattering models for the remote sensing of ice clouds. Part 2: Narrowband models, J. Appl. Meteor., 44, 1896-1911, 2005b.

Bréon, F. M. and Goloub, P.: Cloud droplet effective radius from spaceborne polarization measurements, Geophys. Res. Lett., 25, 1879-1882, 1998.

Chepfer, H., Goloub, P., Riedi, J., De Haan, J. F., Hovenier, J. W., and Flamant, P. H.: Ice crystal shapes in cirrus clouds derived from POLDER-1/ADEOS-1, J. Geophys. Res., 106, 7955-7966, 2001. 
De Haan, J. F., Bosma, P. B., and Hovenier, J. W.: The adding method for multiple scattering calculations of polarized light, Astron. Astrophys., 183, 371-391, 1987.

Dubuisson, P., Buriez, J.-C., and Fouquart, Y.: High spectral resolution solar radiative transfer in absorbing and scattering media application to the Satellite Simulation, J. Quant. Spectrosc. Ra., 55, 103-126, 1996.

Dubuisson, P., Giraud, V., Chomette, O., Chepfer, H., and Pelon, J.: Fast radiative transfer modeling for infrared imaging radiometry, J. Quant. Spectrosc. Ra., 95, 201-220, 2005.

Goloub, P., Herman, M., Chepfer, H., Riedi, J., Brogniez, G., Couvert, P., and Séze, G.: Cloud Thermodynamical Phase Classification from the POLDER Spaceborne instrument, J. Geophys. Res., 105, 14747-14759, 2000.

Hahn, C. J., Warren, S. G., London, J., Chervin, R. M., and Jenne, R.: Atlas of simultaneous occurrence of different cloud types over the ocean, NCAR Tech. Note TN-201 STR, 212 pp., 1982.

Hahn, C. J., Warren, S. G., London, J., Chervin, R. M., and Jenne, R.: Atlas of simultaneous occurrence of different cloud types over land, NCAR Tech. Note TN-241 STR, 209 pp., 1984.

Hutchison, K. D., Etherton, B. J., Topping, P. C., and Huang, H. L.: Cloud top phase determination from the fusion of signatures in daytime AVHRR imagery and HIRS data, Int. J. Remote Sens., 18, 3245-3262, 1997.

Key, J. and Intrieri, J.: Cloud particle phase determination with the AVHRR, J. Appl. Meteorol., 39, 1797-1805, 2000.

King, M. D., Platnick, S., Yang, P., Arnold, G. T., Gray, M. A., Riedi, J. C., Ackerman, S. A., and Liou, K. N.: Remote sensing of liquid water and ice cloud optical thickness, and effective radius in the arctic: Application of airborne multispectral MAS data, J. Atmos. Ocean. Tech., 21, 857-875, 2004.

Knap, W., Stammes, P., and Koelemeijer, R. B. A.: Cloud thermodynamic phase determination from near-infrared spectra of reflected sunlight, J. Atmos. Sci., 59, 83-96, 2002.

C.-Labonnote, L., Brogniez, G., Buriez, J. C., Doutriaux-Boucher, M., Gayet, J. F., and Macke, A.: Polarized light scattering by inhomogeneous hexagonal monocrystals. Validation with ADEOSPOLDER measurements, J. Geophys. Res., 106, 12139-12153, 2001.

Lee, J., Yang, P., Dessler, A. E., Baum, B. A., and Platnick, S.: The influence of thermodynamic phase on the retrieval of mixedphase cloud microphysical and optical properties in the visible and near-infrared region, IEEE Trans. Geoscience and Remote Sensing Letters, 3, 287-291, 2006.

Moody, E. G., King, M. D., Platnick, S., Schaaf, C. B., and Gao, F.: Spatially complete global spectral surface albedos: Valueadded datasets derived from Terra MODIS land products, IEEE T. Geosci. Remote, 43, 144-158, 2005.

Nasiri, S. L. and Baum, B. A.: Daytime multilayered cloud detection using multispectral imager data, J. Atmos. Ocean. Tech., 21, 1145-1155, 2004.

Parol, P., Buriez, J. C., Vanbauce, C., Riedi, J., Labonnote, L. C., Doutriaux-Boucher, M., Vesperini, M., Sèze, G., Couvert, P., Viollier, M., and Br'eon, F. M.: Capabilities of multi-angle polarization cloud measurements from satellite: POLDER results, Adv. Space Res., 33, 1080-1088, 2004.
Pavolonis, M. J. and Heidinger, A. K. Daytime cloud overlap detection from AVHRR and VIIRS, J. Appl. Meteorol., 43, 762-778, 2004.

Pavolonis, M. J., Heidinger, A. K., and Uttal, T.: Daytime global cloud typing from AVHRR and VIIRS: Algorithm description, validation, and comparisons, J. Appl. Meteorol., 44, 804-826, 2005.

Pilewskie, P. and Twomey, S.: Cloud phase discrimination by reflectance measurements near 1.6 and $2.2 \mu \mathrm{m}$, J. Atmos. Sci., 44, 3410-3420, 1987.

Platnick, S., King, M. D., Ackerman, S. A., Menzel, W. P., Baum, B. A., Riedi, J., and Frey, R. A.: The MODIS Cloud Products : Algorithms and Examples from Terra, IEEE T. Geosci. Remote, 41, 459-473, 2003.

Riedi, J.: Analysis of cloud thermodynamic phase at global scale using polarimetric multiangle measurements from the POLDER1/ADEOS1, Ph.D. thesis, Laboratoire d'Optique Atmosphérique, Université des Sciences et Technologies de Lille, 2001.

Riedi, J., Goloub, P., and Marchand, R. T.: Comparison of POLDER cloud phase retrievals to active remote sensors measurements at the ARM SGP site, Geophys. Res. Lett., 28, 2185 2188, 2001.

Seemann, S. W., Borbas, E. E., Knuteson, R. O., Stephenson, G. R., and Huang, H.-L.: Development of a Global Infrared Land Surface Emissivity Database for Application to Clear Sky Sounding Retrievals from Multi-spectral Satellite Radiance Measurements, J. Appl. Meteor. Climatol., 47, 108-123, 2008.

Strabala, K. I., Ackerman, S. A., and Menzel, W. P.: Cloud Properties inferred from 8-12- $\mu$ m Data, J. Appl. Meteor., 33, 212-229, 1994.

Takano, Y., Liou, K. N., and Minnis, P.: The Effects of Small Ice Crystals on Cirrus Infrared Radiative Properties, J. Atmos. Sci., 49, 1487-1493, 1992.

Tian, L. and Curry, J. A.: Cloud overlap statistics, J. Geophys. Res., 94, 9925-9935, 1989.

Tremblay, A. and Glazer, A.: An Improved Modeling Scheme for Freezing Precipitation Forecasts, Mon. Weather Rev., 128(5), 1289-1308, 2000.

Vanbauce, C., Buriez, J. C., Parol, F., Bonnel, B., Sèze, G., and Couvert, P.: Apparent pressure derived from ADEOS-POLDER observations in the oxygen A-band over ocean, Geophys. Res. Lett., 25, 3159-3162, 1998.

Vanbauce, C., Cadet, B., and Marchand, R. T.: Comparison of POLDER apparent and corrected oxygen pressure to ARM/MMCR cloud boundary pressures, Geophys. Res. Lett., 30, 16.1-16.4, 2003.

Yang, P. and Liou, K. N.: Light Scattering and Absorption by Nonspherical Ice Crystals, in Light Scattering Reviews: Single and Multiple Light Scattering, edited by: Kokhanovsky, A. A., Springer-Praxis, 31-71, 2006. 